\title{
Dilepton production and the onset of deconfinement
}

\author{
Enrico Scomparin* \\ INFN - Torino (Italy) \\ E-mail: scompar@to.infn.it
}

Dileptons are a very important tool for the study of the strongly interacting matter created in ultrarelativistic heavy-ion collisions. In particular, the charmonium resonances, experimentally detected through their decay into lepton pairs, are expected to be sensitive to the temperature of the medium, their production being suppressed by color screening when the deconfinement conditions are reached. In this paper we review the studies performed at SPS energy, with an emphasis on the most recent results obtained by the NA60 collaboration.

Critical Point and Onset of Deconfinement

July 3-6 2006

Florence, Italy

${ }^{*}$ Speaker. 


\section{Introduction, results from past experiments}

The study of dilepton production in heavy-ion collisions at the CERN SPS provides some of the most interesting observables explored so far in the search for the quark-gluon plasma. In particular $\mathrm{J} / \psi$ suppression is one of the most important signatures for the formation of a deconfined phase [1]. The NA38 and NA50 experiments have extensively studied $\mathrm{J} / \psi$ production, through its dilepton decay, in various colliding systems, including p-A, $\mathrm{S}-\mathrm{U}$ and $\mathrm{Pb}-\mathrm{Pb}[2,3,4,5]$. Proton-nucleus data provide an important reference, which describes the expected absorption for $\mathrm{J} / \psi$ crossing cold nuclear matter. In particular, the cross section for $\mathrm{J} / \psi$ production has been measured by means of 400 and $450 \mathrm{GeV}$ proton beams incident on up to 7 nuclear targets. In Fig. 1 the J/ $\psi$ production cross section, divided by the target mass number $A$, is shown as a function of $A$. Data have been analyzed in the frame of the Glauber model and in this way it has been possible to calculate the absorption cross section (also known as "normal nuclear absorption") of the $c \bar{c}$ pair in nuclear matter. A simultaneous analysis of the data sets shown in Fig. 1 allows to extract $\sigma_{a b s}^{J / \psi}=4.18 \pm 0.35$ $\mathrm{mb}$. By using the Glauber model it is then possible to calculate the expected contribution of normal nuclear absorption for nucleus-nucleus collisions, as a function of the centrality of the interaction. However, since nucleus-nucleus data were taken at $200 \mathrm{GeV} /$ nucleon (S-U) and $158 \mathrm{GeV} /$ nucleon $(\mathrm{Pb}-\mathrm{Pb})$, a considerably lower energy with respect to $\mathrm{p}-\mathrm{A}$, it is also necessary to take into account in the calculation the $\sqrt{s}$ dependence of the $\mathrm{J} / \psi$ production cross sections. The low statistics $\mathrm{p}-\mathrm{A}$ data points, taken at $200 \mathrm{GeV}$ and also shown in Fig. 1, obviously do not help to constrain $\sigma_{a b s}^{J / \psi}$, but are useful to constrain the absolute normalization of the absorption curve.

In Fig. 2, the continuous line shows the expected $\mathrm{J} / \psi$ yield, at $158 \mathrm{GeV} /$ nucleon, as a function of centrality, expressed through $L$, the mean thickness of nuclear matter crossed by the $c \bar{c}$ pair in its way through projectile and target nucleons. More in detail, the yield shown in Fig. 2 refers to the ratio $\mathrm{J} / \psi / \mathrm{DY}$. This quantity, being the ratio of two experimentally accessible quantities, has the advantage of being free from systematical errors connected with the estimate of efficiencies and luminosities.

Then, by comparing the centrality dependence of the $\mathrm{J} / \psi$ yield observed in A-A collisions to the calculated reference, one can look for an extra-suppression (usually referred to as "anomalous" $\mathrm{J} / \psi$ suppression) that might be connected with the color screening expected at the onset of deconfinement. By looking at the data of Fig. 2 one can see that S-U collisions nicely follow the pattern expected from normal nuclear absorption. On the contrary, for $\mathrm{Pb}-\mathrm{Pb}$ collisions, above a certain threshold, an anomalous suppression sets in, and the $\mathrm{J} / \psi$ yield becomes considerably lower than expected from the nuclear absorption scenario [6].

The observations done by NA38/NA50 have led to a lively and intense debate on the origin of the anomalous $\mathrm{J} / \psi$ suppression. Between the various physics mechanisms investigated by theory, one can mention the onset of a percolation transition [7], the dissociation of charmonia resonances in a Quark-Gluon Plasma [8] and the contribution of a hot gas of hadronic resonances [9, 10].

The extreme interest of this physics topics clearly deserves further experimental studies, and in particular it is important to collect other sets of accurate measurements, obtained with different collision systems. The NA60 experiment at the CERN SPS, with an innovative experimental apparatus improving the dilepton detection technique, studies $\mathrm{J} / \psi$ production in In-In collisions, in order to investigate if the suppression is already present in systems lighter than $\mathrm{Pb}-\mathrm{Pb}$. The com- 


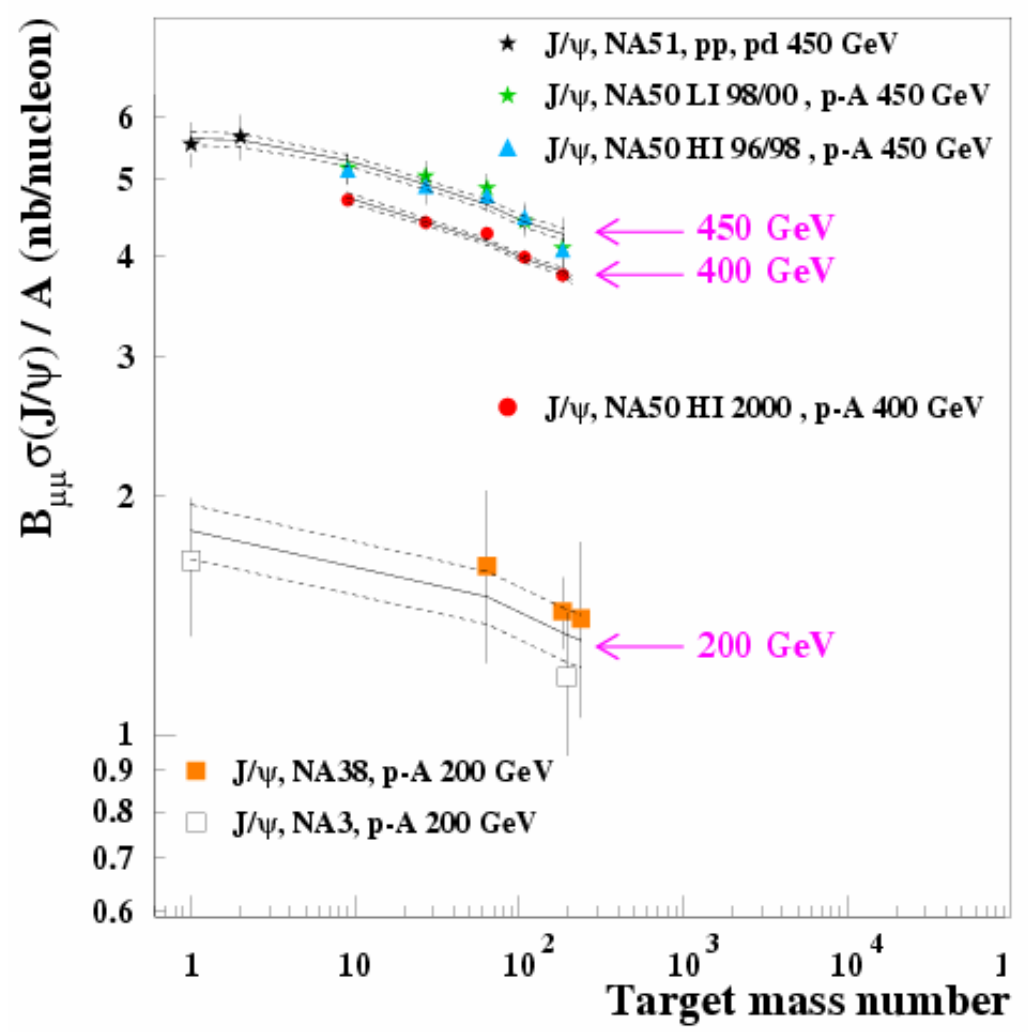

Figure 1: $\mathrm{J} / \psi$ production cross sections measured in $\mathrm{p}-\mathrm{A}$ collisions at SPS energies.

parison between different colliding systems should also allow to understand which is the physics variable at the origin of the $\mathrm{J} / \psi$ suppression.

\section{The NA60 experiment, apparatus and results}

The NA60 experimental apparatus is based on a muon spectrometer, inherited from the NA50 experiment, complemented by a new target region, based on a Beam Tracker and a Vertex Tracker placed in a $2.5 \mathrm{~T}$ dipole magnet. The target system is made of $7 \mathrm{In}$ targets, $1.5 \mathrm{~mm}$ thick each, placed in vacuum, corresponding to a total interaction probability of $\sim 20 \%$. A Zero Degree Calorimeter (ZDC) provides an estimate of the centrality of the collisions, by measuring the energy $\left(E_{Z D C}\right)$ released by the projectile nucleons which have not taken part in the interaction. A more detailed description of the apparatus can be found in $[11,12]$. The reconstruction of the muon tracks in the muon spectrometer is affected by multiple scattering and energy loss fluctuations due to the crossing of the muons through the hadron absorber. To overcome this limitation, NA60 measures muons before their entrance into the hadron absorber, by means of the vertex tracker. Muon tracks measured in the muon spectrometer are matched with tracks reconstructed by the vertex tracker, resulting in an improvement of the dimuon mass resolution and in an accurate determination of the muons' origin. Details on the matching technique can be found in [13]. 


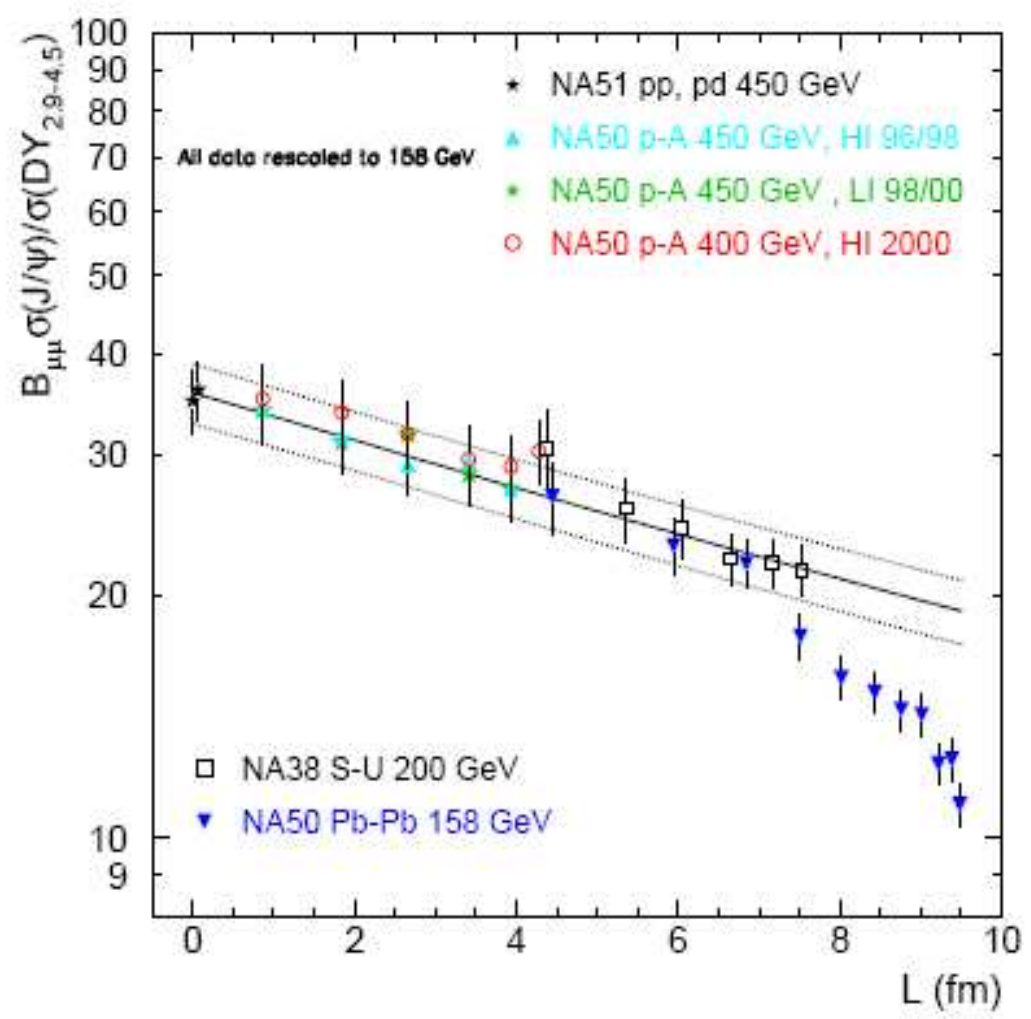

Figure 2: The ratio J/ $\psi$ / DY, for proton-nucleus and nucleus-nucleus collisions, at SPS energies.

The sample of nucleus-nucleus data collected by NA50 consists of $\sim 230$ million dimuon triggers, obtained studying In-In collisions at $158 \mathrm{GeV} /$ nucleon. Two muon spectrometer settings have been used during the data taking, corresponding to two values of the magnet current: 4000 $A$ and $6500 \mathrm{~A}$. Events collected with the lower current have a better acceptance for the low mass dimuons, while the higher current setting improves the mass resolution of the $J / \psi$. The analysis shown here includes both data sets, i.e. the whole statistics collected by the NA60 experiment.

The event selection criteria have been tuned in order to obtain a clean sample of $J / \psi$ events produced in primary In-In collisions. Tracks are reconstructed in the vertex tracker and it is required to have at least one vertex satisfying various quality cuts, including a minimum number of tracks $(\geq 4)$ attached to it. The longitudinal coordinate of that vertex, which is reconstructed with an accuracy of $\sim 200 \mu \mathrm{m}$, must lie inside one of the seven targets. The matching of the muon tracks makes it possible to determine the longitudinal coordinate of the dimuon production point with a precision of few hundreds $\mu \mathrm{m}$. Asking the dimuon vertex to coincide with the most upstream interaction vertex in the target region ensures that the $\mathrm{J} / \psi$ is produced in a primary In-In collision. A further set of selections is done in order to minimize the event pile-up in the apparatus. It is based on the good timing properties (a few ns) and on the excellent granularity of the Beam Tracker, that allows to reject events with several ions arriving close in time. This selection is particularly important for obtaining an unbiased $E_{Z D C}$ distribution. In fact, since the ZDC is placed on the beam axis and records a signal for each incoming ion, it is particularly sensitive to this 
kind of background. Finally, in order to reject events coming from the edges of the spectrometer acceptance, only events having $0<y_{c m s}<1$ and $-0.5<\cos \theta_{C S}<0.5$ have been used. $\theta_{C S}$ is the polar angle of the muons in the Collins-Soper reference frame.

Two complementary analysis have been performed in order to study the centrality dependence of the $\mathrm{J} / \psi$ production in Indium-Indium collisions. They differ in the way used for normalizing the $\mathrm{J} / \psi$ distributions. The first technique, the so-called "standard" analysis, is based on the normalization of the J/ $\psi$ to the Drell-Yan (DY) yield. In this approach, already used by the NA38/NA50 experiments, Drell-Yan is used as a reference process. This choice allows a significant reduction of the systematic errors, as already outlined in Section 1. Furthermore, being independent from final state effects, the DY production cross section scales with the number of nucleon-nucleon collisions and represents, therefore, an ideal normalization for a hard process, like $\mathrm{J} / \psi$ production. In the "standard" analysis, the ratio between the $\mathrm{J} / \psi$ and the DY yields is extracted by fitting the opposite sign dimuon mass spectrum to a superposition of different contributions. Above $2 \mathrm{GeV} / \mathrm{c}^{2}$, the dimuon invariant mass region contains the $\mathrm{J} / \psi$ and the $\psi^{\prime}$ resonances, an underlying continuum composed of Drell-Yan events and semi-muonic decays of $D$ and $\bar{D}$ mesons, and a combinatorial background from $\pi$ and $K$ decays. The expected mass shapes of the signals are evaluated through a Monte Carlo simulation based on Pythia with GRV94LO parton distribution functions. These simulations also provide the values of the $\mathrm{J} / \psi$ and DY acceptances $\left(A_{\mathrm{J} / \psi}=12.4 \%, A_{D Y(2.9-4.5)}=13.0 \%\right.$ for the data collected with the current of $6500 A$ in the muon spectrometer and slightly larger for the $4000 \mathrm{~A}$ ). In Fig. 3 the fit of the invariant mass spectrum is shown for the $6500 \mathrm{~A}$ data sample, together with the contributions of the various physics sources.

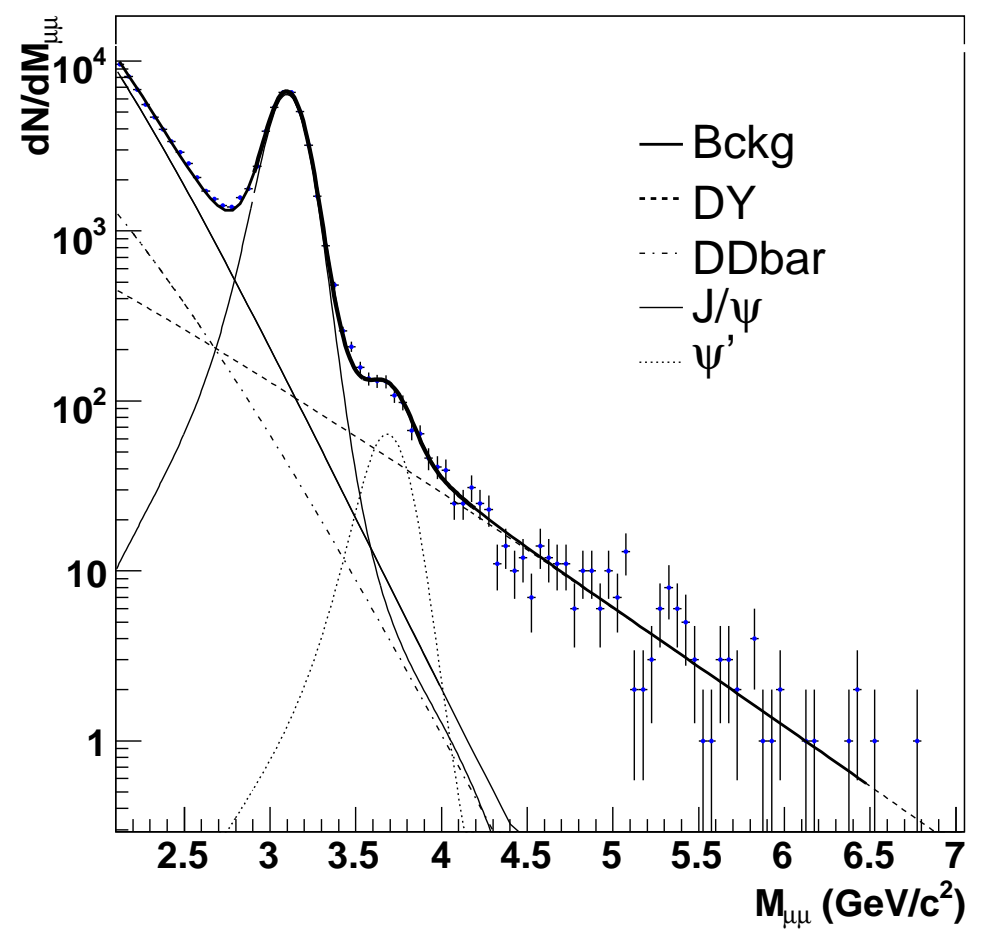

Figure 3: Fit of the $\mu^{+} \mu^{-}$invariant mass spectrum, for the $6500 \mathrm{~A}$ data sample. 
The combinatorial background has been estimated from the measured sample of like-sign pairs. Results obtained from the two sets of data, corresponding to the two configurations of the muon spectrometer, are found to be statistically compatible. Therefore the results shown in the following are based on their average. Although the use of DY as a reference process has many advantages, it induces a large statistical error in the results, due to the limited number of high mass DY. Therefore, in order to increase the statistics as much as possible, the matching of the muon candidates in the vertex spectrometer has not been required. This allows to increase the statistics by $\sim 40 \%$, for a total of $\sim 320$ DY events with mass above $4.2 \mathrm{GeV} / \mathrm{c}^{2}$ and $\sim 45000 \mathrm{~J} / \psi$. In this case a further cut based on the extrapolation of the muon spectrometer tracks ensures that the dimuon comes from the target region. In spite of the statistics gain, the number of high mass Drell-Yan events is still limited; as a consequence the $\mathrm{J} / \psi / \mathrm{DY}$ ratio cannot be studied in more than three centrality bins. The $\mathrm{J} / \psi / \mathrm{DY}$ values have then been compared to expectations from normal nuclear absorption, using the results obtained by NA50 and detailed in Section 1. In Fig. 4 the measured $\mathrm{J} / \psi / \mathrm{DY}$ ratios have been divided out by the values expected in case of nuclear absorption, and have been plotted as a function of the number of participants $\left(\mathrm{N}_{\text {part }}\right) ; \mathrm{S}-\mathrm{U}$ and $\mathrm{Pb}-\mathrm{Pb}$ results are also shown. The centrality has been determined using $E_{Z D C}$ and taking into account the smearing induced by the detector resolution. We see that a suppression of the $\mathrm{J} / \psi$ beyond nuclear absorption is present in In-In collisions. However, the small number of bins prevents an accurate study of the $\mathrm{J} / \psi$ centrality dependence as well as a meaningful comparison with the results obtained by NA38/NA50.

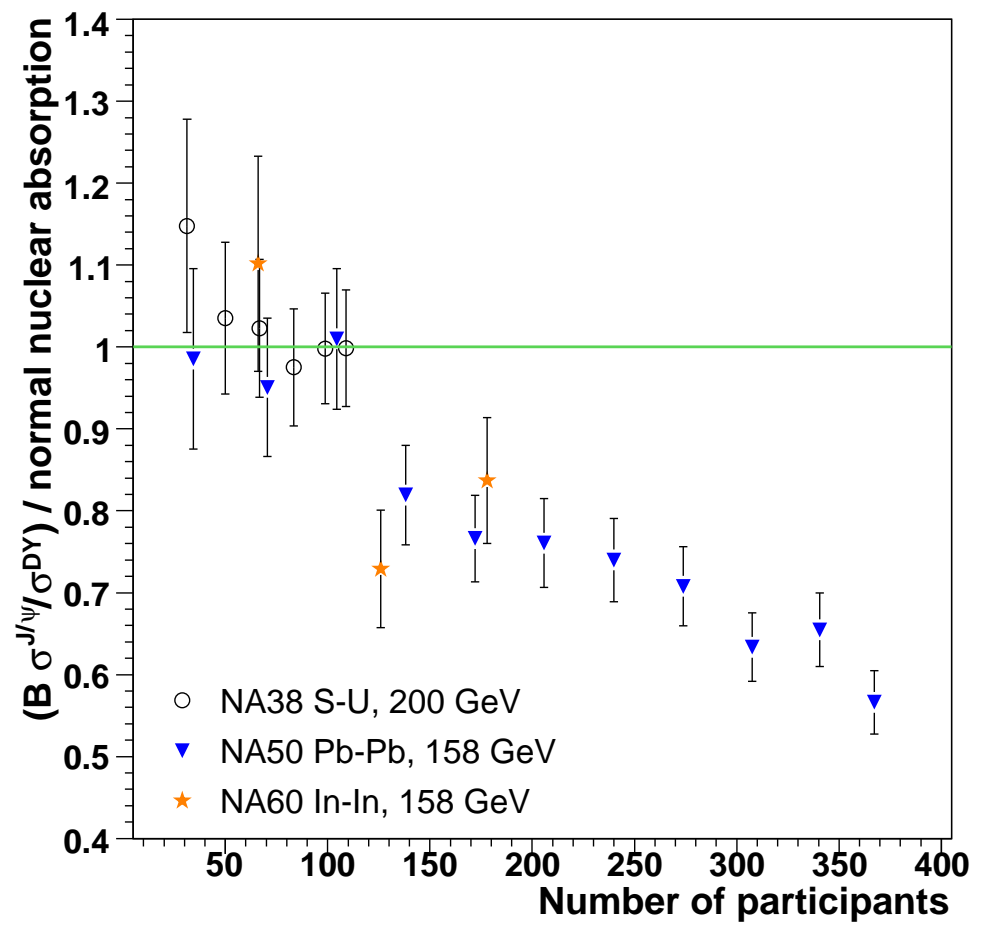

Figure 4: J/ $\psi /$ DY, divided by the normal nuclear absorption curve, as a function of $\mathrm{N}_{\text {part }}$, for S-U, In-In and $\mathrm{Pb}-\mathrm{Pb}$ collisions. 
The second analysis technique overcomes the problem of the low Drell-Yan statistics by directly studying the measured $\mathrm{J} / \psi$ centrality distribution as a function of $E_{Z D C}$. Following this approach, the $\mathrm{J} / \psi$ sample is not normalized to a measured quantity. Rather, the observed $\mathrm{J} / \psi$ centrality distribution is directly compared to the theoretical distribution expected in case nuclear absorption is the only suppression mechanism. Since the number of $J / \psi$ is not normalized to other data collected under the same conditions, one has to check that no centrality-dependent inefficiencies are introduced by the selection criteria and, more generally, that systematic errors are reduced as much as possible. For this reason in this analysis the matching of the muon tracks is required, in order to identify and discard events where the $\mathrm{J} / \psi$ is not produced in primary In-In collisions. It has been checked, by means of a Monte Carlo simulation, that the matching efficiency as a function of centrality for $\mathrm{J} / \psi$ events increases by less than $2 \%$ from central to peripheral collisions, inducing a negligible bias on the centrality distribution of the measured J/ $\psi$. In addition, the matching improves significantly the $\mathrm{J} / \psi$ mass resolution, from 105 to $70 \mathrm{MeV}$, and reduces the combinatorial background from 3 to $1 \%$ in the corresponding mass region. It has also been checked that the vertexing efficiency for events where a $\mathrm{J} / \psi$ has been produced, decreases by less than $1 \%$ from central to peripheral collisions. After event selection, the $\mathrm{J} / \psi$ distribution as a function of centrality has been obtained by means of a simple fitting procedure that allows to subtract the small amount of Drell-Yan and combinatorial background under the resonance peak. The $\mathrm{J} / \psi$ centrality distribution is then compared to expectations from pure nuclear absorption. The relative normalization between data and the reference curve is not fixed a priori; therefore the ratio between data and the nuclear absorption curve, integrated over centrality, is assumed to be the same as in the "standard" J/ $\psi /$ DY analysis, i.e. $0.87 \pm 0.05$.

Due to the high $\mathrm{J} / \psi$ statistics this approach provides results with negligible statistical errors. A careful study of systematic errors is therefore mandatory. Their most important source is connected with the determination of the nuclear absorption curve and with the determination of $\mathrm{N}_{\text {part }}$ starting from $E_{Z D C}$. The calculation of the nuclear absorption curve is based on the Glauber model. Therefore the influence of the choice of the inputs on the result has been investigated. Different Indium nuclear density distributions [14] with respect to the default parameterization [15] have been tested. This choice does not affect significantly the shape of the absorption curve, since the effect is smaller than $1 \%$ for $E_{Z D C}>3 \mathrm{TeV}$. For very central events the effect is larger and reaches $\sim 15 \%$. Concerning the centrality determination, the zero degree energy is well correlated with $\mathrm{N}_{\text {part }}$. However, the ZDC does not measure only spectator nucleons but also a small amount of energy released by the forward secondary particles, emitted in the calorimeter's acceptance $(\eta>6.3)$. The uncertainty on this contribution, important only for very central collisions, has been conservatively assumed to be of the order of $10 \%$. Such an uncertainty gives, for events with $E_{Z D C}<3 \mathrm{TeV}$, a systematic error of the order of $9 \%$ on the absorption curve. For more peripheral events the effect becomes quickly negligible. Furthermore, the calculation of the absorption curve depends on $\sigma_{J / \psi}^{p p}$ and on $\sigma_{J / \psi}^{a b s}$, both estimated, as explained in Section 1, from p-A collisions at 450 and $400 \mathrm{GeV}$ by NA50. The experimental errors on such values, and in particular the factors needed to rescale $\sigma_{J / \psi}^{p p}$ to $158 \mathrm{GeV}$, induce a systematic error on the absorption curve of the order of $8 \%$, almost independent of centrality. In the near future, results from a p-A data sample collected by NA60 at $158 \mathrm{GeV}$ and presently being analyzed, should help to reduce significantly this uncertainty. Fi- 
nally, since data have been normalized to the absorption curve using the results from the "standard" analysis, the $6 \%$ error related to the uncertainty in the normalization factor has also to be taken into account. Combining the various sources, one ends up with a $\sim 11 \%$ systematic error, independent of centrality. On top of that, the most central bins are affected by a further, sizeable systematic error relatively to the others. In Fig. 5 the ratio between the measured number of $\mathrm{J} / \psi$ events and the nuclear absorption curve is shown as a function of $\mathrm{N}_{\text {part }}$. The various sources of systematic errors are also shown. It can be seen that an anomalous suppression sets in for semiperipheral In-In collisions $\left(\mathrm{N}_{\text {part }} \sim 80\right)$ and that the effect saturates for more central events.

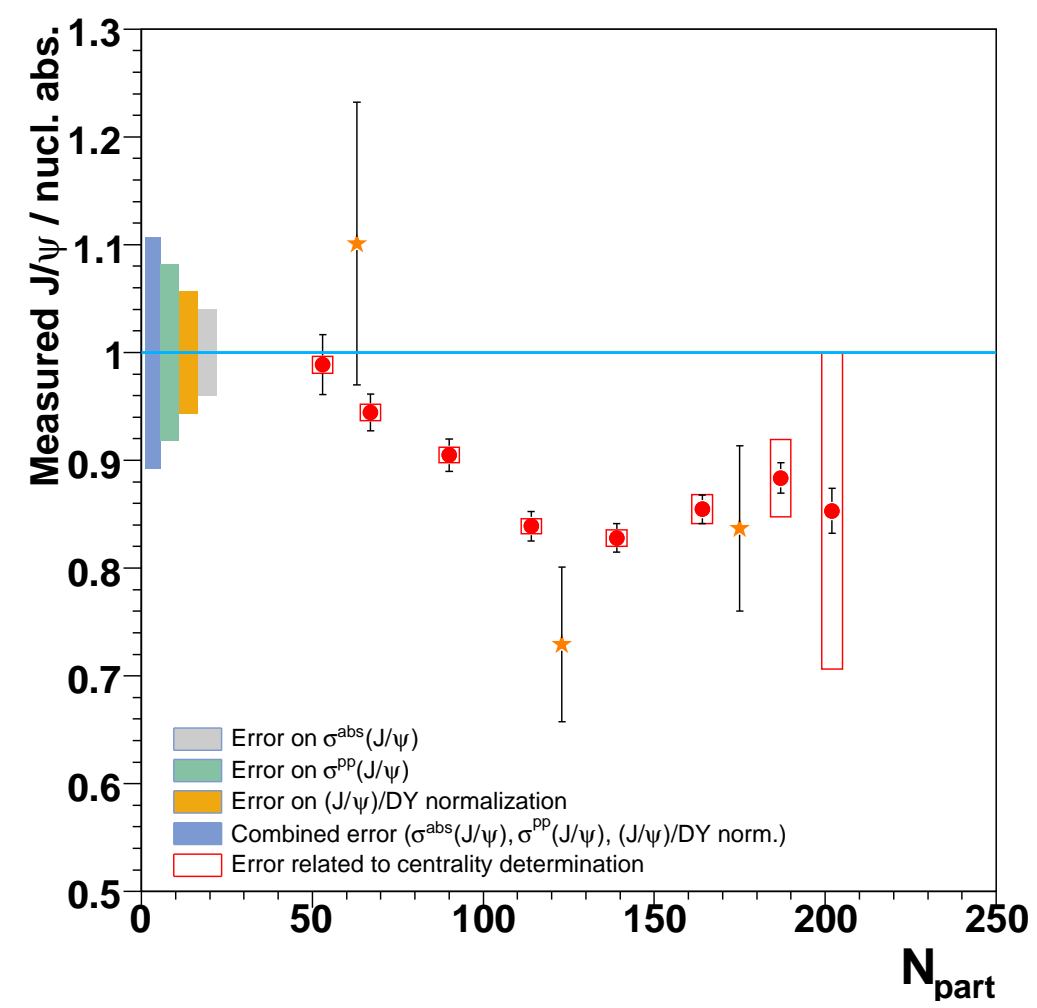

Figure 5: Measured $\mathrm{J} / \psi$ yield, divided by the normal nuclear absorption curve, as a function of $\mathrm{N}_{\text {part }}$, for In-In collisions.

In Fig. 6 the results of this analysis are compared with previous results obtained by NA38 and NA50. Although this comparison must be considered as preliminary, due to the possible systematics connected with the use of different centrality estimators (transverse energy for NA38/NA50, $E_{Z D C}$ for NA60), a fair agreement between the various systems can be observed. It should be noted that the systematic errors related to the energy rescaling of the absorption curve affect in the same way the points measured by NA38, NA50 and NA60. Therefore they do not influence the relative positions of the patterns observed in $\mathrm{S}-\mathrm{U}$, In-In and $\mathrm{Pb}-\mathrm{Pb}$.

Finally, NA60 has also obtained preliminary azimuthal angle distributions of the J/ $\psi$ produced in In-In collisions. These distributions have been obtained with respect to the orientation of the reaction plane, defined by the beam direction $\vec{z}$ and the impact parameter vector $\vec{b}$. This plane is experimentally not directly accessible, but it can be determined from the emission angles of the charged particles measured in the vertex tracker, using the so-called event plane method. The 


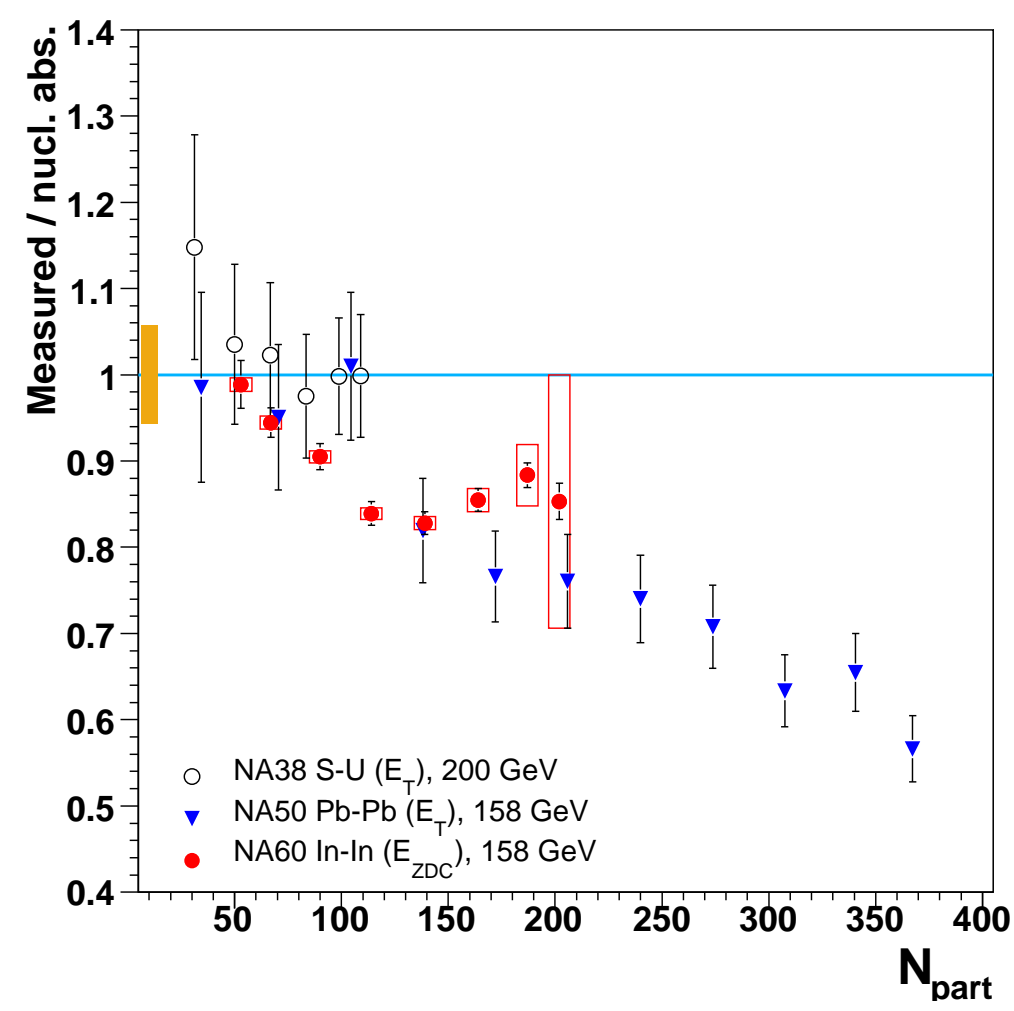

Figure 6: Comparison between the suppression patterns observed in $\mathrm{S}-\mathrm{U}$, In- $\mathrm{In}$ and $\mathrm{Pb}-\mathrm{Pb}$ collisions, as a function of $\mathrm{N}_{\text {part }}$.

$\mathrm{J} / \psi$ anisotropic flow is then quantified evaluating the coefficients of a Fourier expansion of the azimuthal distribution. The azimuthal angle distribution for $\mathrm{J} / \psi$ is shown in Fig. 7 for central $(0.5<$ $\left.\sigma / \sigma_{\text {geo }}<20 \%\right)$ and peripheral events $\left(20<\sigma / \sigma_{\text {geo }}<83 \%\right)$. The elliptic flow coefficient $\mathrm{v}_{2}$ is obtained after correcting for the event plane resolution. The distributions are based on $\sim 12000 \mathrm{~J} / \psi$, which is about $\sim 50 \%$ of the full statistics available for this kind of analysis. The more peripheral data seem to indicate a non-isotropic emission pattern but the limited statistics does not allow, for the moment, strong conclusions. A detailed review of this analysis is reported in [16].

\section{Conclusions}

The analysis of the data taken by NA60 in In-In collisions shows that the $J / \psi$ is anomalously suppressed. An analysis method based on the comparison of the $\mathrm{J} / \psi$ centrality distribution with the expectation from a pure nuclear absorption scenario shows that the anomalous suppression sets in at $\mathrm{N}_{\text {part }} \sim 80$, with a saturation for more central events. Such an onset roughly corresponds to that already observed for $\mathrm{Pb}-\mathrm{Pb}$ collisions. The statistical errors on this analysis are negligible (of the order of $2 \%$ ), while there is a $10 \%$ systematic error, independent of centrality and therefore not affecting the shape of the $\mathrm{J} / \psi$ suppression pattern. Most of the systematic errors come from the uncertainty in the knowledge of the $\mathrm{J} / \psi$ nuclear absorption, which has been accurately measured at $450 \mathrm{GeV}$, but not at $158 \mathrm{GeV}$, the energy of the heavy-ion data taking. The situation is expected 

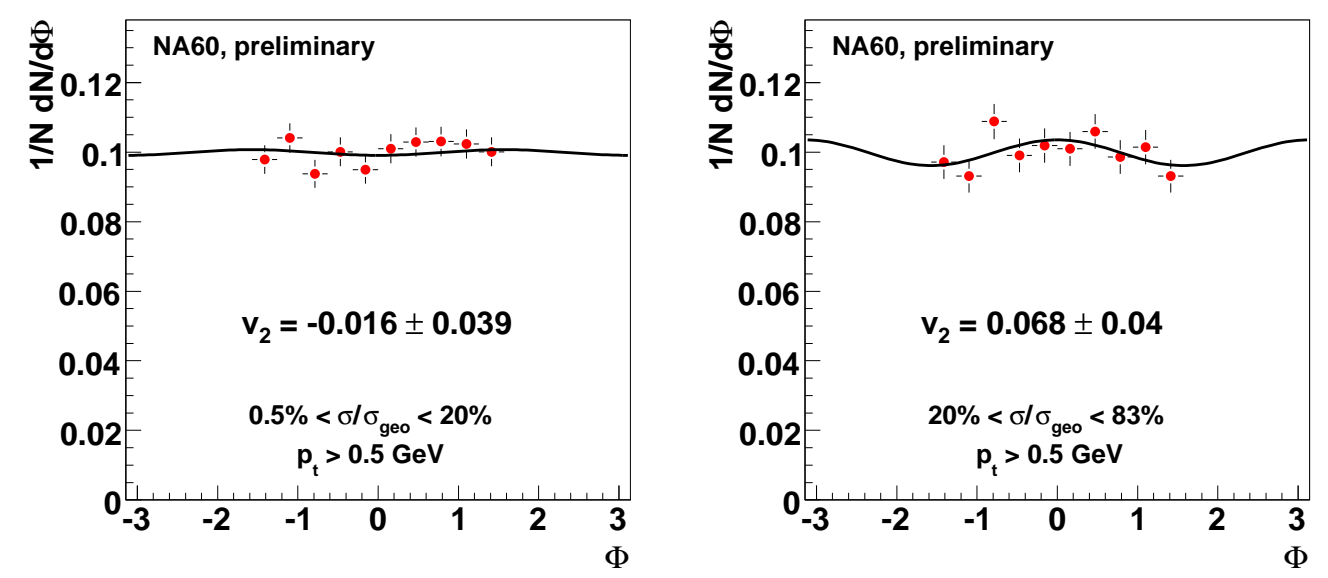

Figure 7: J/ $\psi$ azimuthal distributions measured in In-In collisions, for two centrality bins.

to improve as soon as results from the analysis of the p-A data collected by NA60 at $158 \mathrm{GeV}$ will be available.

\section{References}

[1] T. Matsui and H. Satz, Phys. Lett. B178 (1986) 416

[2] B. Alessandro et al. (NA50 Coll.), Eur. Phys. J. C39 (2005) 335

[3] M.C. Abreu et al. (NA38 Coll.), Phys. Lett. B449 (1999) 128

[4] B. Alessandro et al. (NA50 Coll.), Phys. Lett. B553 (2003) 167

[5] B. Alessandro et al. (NA50 Coll.), Eur. Phys. J. C33 (2004) 31

[6] L. Ramello et al. (NA50 Coll.), Nucl. Phys. A774 (2006) 59

[7] S. Digal, S. Fortunato and H. Satz, Eur. Phys. J. C32 (2004) 547

[8] R. Rapp, Eur. Phys. J. C43 (2005) 91

[9] A. Capella and E. Ferreiro, Eur. Phys. J. C42 (2005) 419

[10] F. Becattini et al., Phys. Lett. B632 (2006) 233

[11] G. Usai et al. (NA60 Coll.), Eur. Phys. J C43 (2005) 415

[12] M. Keil et al., Nucl. Instrum. Meth. A539 (2005) 137 and A546 (2005) 448

[13] R. Shahoyan et al (NA60 Coll.), Nucl. Phys. A774 (2006) 677

[14] Landolt-Börnstein, Numerical Data, Springer-Verlag, 1967

[15] De Vries et al., Atomic Data and Nuclear Data Tables 36, (1987) 495

[16] A. Förster et al. (NA60 Coll.), to appear in the proceedings of Strangeness in Quark Matter, March 26-31, 2006 